\section{Cannabis und Psychose - ein kausaler Zusammenhang?}

Eine Reihe von Studien hat nahegelegt, dass Cannabiskonsum das Risiko für Psychosen erhöht und dass eventuell sogar ein Dosis-Wirkungs-Zusammenhang besteht.

$\mathrm{D}$ ie hier vorgestellte Metaanalyse untersuchte den potenziellen Zusammenhang zwischen Cannabiskonsum und dem Risiko für Psychosen anhand einer zusammenfassenden Auswertung einer Reihe von Studien.

Eingeschlossen wurden zehn Studien, die insgesamt 66.816 Personen umfassten. Eindeutig konnte festgestellt werden, dass ein deutlicher Cannabiskonsum das Risiko, eine Psychose zu entwickeln, nahezu um den Faktor 4 (Odds ratio [OR]: 3,90, $95 \%$-KI: 2,84; 5,34]) erhöht, selbst ein mäßiger Cannabiskonsum führte noch zu einer Verdoppelung des Risikos (OR: 1,97, 95\%-KI: 1,68; 2,31 ). Auch eine Dosis-Wirkungsbezie- hung konnte gezeigt werden. Die Autoren verweisen in der Diskussion zusätzlich auf andere Studien, die dargelegt hätten, dass der Konsum hochpotenter Cannabinoide und auch der frühzeitige Beginn des Cannabiskonsums - neben der Dosis - zusätzliche Risikofaktoren für eine Psychose darstellten.

\section{Kommentar}

Richtigerweise - und dies wird bei solchen letztendlich korrelativen Studien häufig vergessen - kann selbst aufgrund einer so ausgeprägten Risikoerhöhung durch einen Faktor (Cannabiskonsum) keine kausale Beziehung zwischen Cannabiskonsum und Psychose etabliert werden. Hierzu sind an-

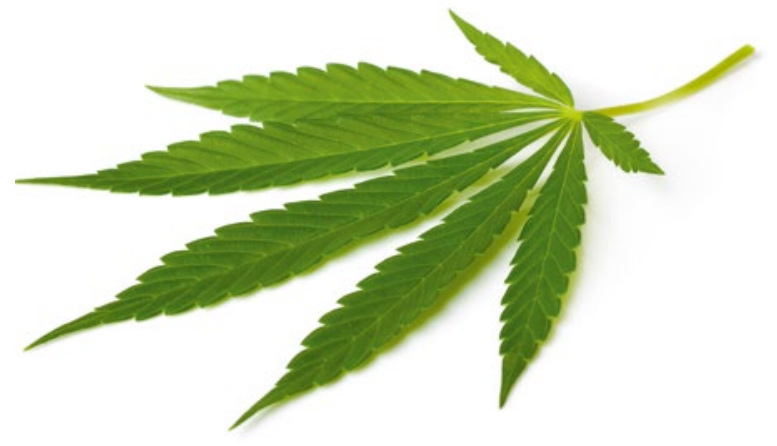

dere wissenschaftliche Untersuchungsansätze notwendig. Allerdings kann dieses Ergebnis durchaus rechtfertigen, Patienten intensiv über das erhöhte Psychoserisiko bei dem Gebrauch von Cannabis aufzuklären, insbesondere wenn zusätzliche Risikofaktoren (wie etwa Psychose bei nahen Verwandten oder bereits bestehende Frühsymptome einer Psychose) vorliegen.

Prof. Dr. med. Hans-Peter Volz

Marconi A, Di Forti M, Lewis CM, Murray RM, Vassos E. Meta-analysis of the Association Between the Level of Cannabis Use and Risk of Psychosis. Schizophr Bull 2016; 42: 1262 -9

\title{
Mortalität bei Psychosen vergrößert sich nicht durch Cannabis
}

\section{Die Mortalität von Patienten mit schizophrenen Psychosen ist im Vergleich zur Allgemeinbevölkerung erhöht. Eine Studie untersuchte, ob sich diese durch fortgesetzten Cannabiskonsum noch weiter erhöht.}

C annabiskonsum wirkt sich auf die Gesundheit von Adoleszenten aus und hierbei sind auch kognitive und psychologische Bereiche betroffen. AuBerdem scheint eine Assoziation zwischen Cannabiskonsum und nicht affektiven Psychosen zu bestehen. Die Mortalität von Patienten mit solchen Psychosen, einschließlich schizophrener Psychosen, ist im Vergleich zur Allgemeinbevölkerung um den Faktor 2 bis 3 erhöht. Der fortgesetzte Konsum von Cannabis bei Psychoserkrankten erhöht das Risiko fortwährender psychotischer Symptome, einer schlechteren Adhärenz und das Rückfallrisiko. Um diese Einzelbeobachtungen an einer großen Kohorte abzusichern, erhob die Autorengruppe
1. das Gesamtrisiko von Cannabiskonsumenten zu sterben im Vergleich zu Nichtkonsumenten sowie die Exzessmortalität, falls zusätzlich eine Psychose auftritt,

2. die Mortalität unter Psychoseerkrankten und das Ausmaß der Beeinflussung dieser Mortalität durch Cannabiskonsum und

3. die Interaktion zwischen Cannabiskonsum und dem Auftreten psychotischer Störungen im Hinblick auf die Mortalität.

Hierzu wurden 50.373 männliche schwedische Rekruten (Alter 18 bis 19 Jahre) im Nationalen Sterberegister identifiziert, wenn sie bis zu ihrem 60. Lebensjahr verstorben waren. Mithilfe komplexer Statistiken wurden die oben erwähn- ten Fragestellungen an dieser großen Kohorte untersucht. Zum einen zeigte sich, dass ein hoher Konsum von Cannabis mit einer erhöhten Sterblichkeit in Zusammenhang steht (Hazard Ratio [HR]: 1,4, $95 \%$-KI: 1,1; 1,8). Auch ergab sich eine Exzessmortalität bei Psychoseerkrankten, die aber nicht im Zusammenhang mit Cannabiskonsum stand. Es wurde also keine Interaktion zwischen dem Gebrauch von Cannabis und psychotischen Störungen im Hinblick auf die Mortalität gefunden.

\section{Kommentar}

Diese Studie zeigte insofern nur, dass Cannabiskonsum zu einer erhöhten Sterblichkeit insgesamt führen könnte und dass das Bestehen einer Psychose mit einer Exzessmortalität verbunden ist. Diese erhöhte Mortalität bei Psychoseerkrankten wurde durch den zusätzlichen Konsum von Cannabis allerdings nicht weiter erhöht.

Prof. Dr. med. Hans-Peter Volz

Manrique-Garcia E, Ponce de Leon A, Dalman C, Andréasson S, Allebeck P. Cannabis, Psychosis, and Mortality: A Cohort Study of 50,373 Swedish Men. Am J Psychiatry 2016; 173: 790-8 